\title{
Electron energy relaxation in the presence of magnetic impurities
}

\author{
A. Kaminski and L.I. Glazman \\ Theoretical Physics Institute, University of Minnesota, Minneapolis, MN 55455, USA
}

\begin{abstract}
We study inelastic electron-electron scattering mediated by the exchange interaction of electrons with magnetic impurities, and find the kernel of the corresponding two-particle collision integral. In a wide region of parameters, the kernel $K$ is proportional to the inverse square of the transferred energy, $K \propto J^{4} / E^{2}$. The exchange constant $J$ is renormalized due to the Kondo effect, yielding an additional weak dependence of $K$ on the energies of the colliding electrons. At small energy transfers, the $1 / E^{2}$ divergence is cut off; the cut-off energy is determined by the dynamics of the impurity spins. The obtained results may provide a quantitative explanation of the experiments of Pothier et al. [Phys. Rev. Lett. 79, 3490 (1997)] on anomalously strong energy relaxation in short metallic wires.
\end{abstract}

PACS numbers: 73.23.-b, 72.15.Qm, 72.10.Fk

The effect of magnetic impurities on the electron properties of a metal is drastically different from that of "usual" defects which just violate the translational invariance of the crystalline lattice. The reason for the difference is that a magnetic impurity brings an additional degree of freedom - its spin. If there were no exchange interaction with the itinerant electrons, the ground state of the system would be degenerate with respect to the orientation of impurity spins. Weak exchange interaction allows an itinerant electron to flip its spin in the course of scattering on a magnetic impurity. Such scattering, accounted for even in the lowest-order (Born) approximation, yields an important effect of dephasing of the electron state. Finite dephasing time, in turn, suppresses the interference corrections to the conductivity, thus suppressing the weak localization effect [1].

The higher-order terms in the perturbation theory series for the scattering amplitude reveal one more important phenomenon. It turns out that the amplitude of scattering caused by the exchange interaction increases with lowering the temperature, as opposed to the temperature-independent scattering on a usual impurity. This increase is responsible for the non-monotonic temperature dependence of the resistivity of a metal, the phenomenon called the Kondo effect [2].

Spin exchange between an electron and a magnetic impurity may occur in an act of elastic scattering. Accounting for these spin-exchange elastic processes is sufficient for understanding the dephasing phenomenon [1] and the Kondo effect [3]. However, such processes do not lead to any energy relaxation of electrons. In this paper we demonstrate that magnetic impurities may also mediate energy transfer between electrons. If the energy transfer $E$ is larger than the Kondo temperature $T_{K}$, then the energy relaxation occurs predominantly in two-electron collisions. We derive the kernel $K$ of the corresponding collision integral in the kinetic equation for the distribution function. This kernel depends strongly on the transferred energy, $K \propto J^{4} / E^{2}$. The dependence of $K$ on the energies $\varepsilon_{i}$ of the colliding electrons (measured from the
Fermi level) is relatively weak as long as $\left|\varepsilon_{i}\right| \gg T_{K}$. This dependence comes from the logarithmic in $\left|\varepsilon_{i}\right|$ renormalization of the exchange integral $J$, known from the theory of Kondo effect [2]. At small energy transfers, the $1 / E^{2}$ divergence of the kernel is cut off; the cut-off energy is determined by the dynamics of the impurity spins, which results from their interaction with the Fermi sea.

The motivation for our study comes from the experiment [4.5] where the relaxation of the electron energy distribution function in mesoscopic wires was investigated. It was found that the empirical relation $K \propto 1 / E^{2}$ holds in a substantial interval of energies $E$ for $\mathrm{Au}$ and $\mathrm{Cu}$ wires. The data of Ref. [4, 5] was accurate enough to rule out the direct Coulomb interaction [6], which would yield $K(E) \propto 1 / E^{3 / 2}$, as a source of relaxation.

We describe the metal with magnetic impurities by means of the exchange Hamiltonian:

$$
\begin{aligned}
& \hat{H}=\hat{H}_{0}+\sum_{l} \hat{V}_{l}, \quad \hat{H}_{0} \equiv \sum_{\mathbf{k} \alpha} \xi_{\mathbf{k}} c_{\mathbf{k} \alpha}^{\dagger} c_{\mathbf{k} \alpha}, \\
& \hat{V}_{l} \equiv \sum_{\mathbf{k} \alpha \mathbf{k}^{\prime} \alpha^{\prime}} J e^{i\left(\mathbf{k}-\mathbf{k}^{\prime}\right) \mathbf{r}_{l}}\left(\hat{\mathbf{S}}_{l} \boldsymbol{\sigma}_{\alpha \alpha^{\prime}}\right) c_{\mathbf{k} \alpha}^{\dagger} c_{\mathbf{k}^{\prime} \alpha^{\prime}},
\end{aligned}
$$

where index $l$ labels the magnetic impurities, $\hat{\mathbf{S}}_{l}$ is the spin operator of the $l$-th impurity, $\hat{\mathbf{S}}_{l}^{2}=S(S+1)$, and $\mathbf{r}_{l}$ is its coordinate. Free electron states are labelled by the wave vector $\mathbf{k}$ and spin index $\alpha$. The Pauli matrices are denoted by $\boldsymbol{\sigma} \equiv\left(\sigma^{x}, \sigma^{y}, \sigma^{z}\right)$.

If the concentration $n$ of the impurities is low enough, they can be considered independently. Therefore we will perform our calculations for a single impurity, omitting the impurity index $l$, and then will multiply the resulting expressions for the scattering rate by $n$. In this oneimpurity problem, there is interaction only in $s$ channel, so we will label the participating electron states with scalar index $k$.

In the framework of the exchange Hamiltonian (11), the lowest non-vanishing order of the perturbation theory series in the exchange constant $J$ for the inelastic scattering amplitude is the second order: 


$$
\begin{aligned}
& A\left(k_{1} \sigma_{1}, k_{2} \sigma_{2}, S \rightarrow k_{3} \sigma_{3}, k_{4} \sigma_{4}, S^{\prime}\right) \delta\left(\xi_{k_{1}}+\xi_{k_{2}}-\xi_{k_{3}}-\xi_{k_{4}}\right)= \\
& \left\langle k_{3} \sigma_{3}, k_{4} \sigma_{4}, S^{\prime}\left|-\int_{-\infty}^{\infty} d t \sum_{k \alpha k^{\prime} \alpha^{\prime}} J \hat{\mathbf{S}}(t) \boldsymbol{\sigma}_{\alpha \alpha^{\prime}} c_{k \alpha}^{\dagger}(t) c_{k^{\prime} \alpha^{\prime}}(t) e^{-0|t|} \int_{-\infty}^{t} d t^{\prime} \sum_{p \beta p^{\prime} \beta^{\prime}} J \hat{\mathbf{S}}\left(t^{\prime}\right) \boldsymbol{\sigma}_{\beta \beta^{\prime}} c_{p \beta}^{\dagger}\left(t^{\prime}\right) c_{p^{\prime} \beta^{\prime}}\left(t^{\prime}\right) e^{-0\left|t^{\prime}\right|}\right| k_{1} \sigma_{1}, k_{2} \sigma_{2}, S\right\rangle .
\end{aligned}
$$

In the diagrammatic representation, the amplitude is the sum of the diagram shown in Fig. 11 and the three other diagrams that can be obtained from the diagram of Fig. 1 by the transposition of indices $1 \leftrightarrow 2$ and/or $3 \leftrightarrow 4$. Note that there is no summation over the initial or final spin states of the impurity in Eq. (2). Therefore, the spin lines are not closed, i.e. contrary to Ref. [7] this scattering amplitude cannot be represented in the form of an effective four-electron vortex.

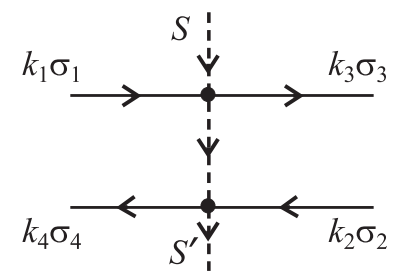

FIG. 1. A characteristic diagram for the amplitude of inelastic electron-electron scattering mediated by the exchange interaction of electrons with a magnetic impurity, in notation of Ref. 8]. The solid lines denote electron states, the dashed lines denote the localized spin state.

Performing the time integrations in Eq. (2), we obtain the standard expression for the second-order term of the expansion of $T$-matrix,

$$
\begin{aligned}
& A\left(k_{1} \sigma_{1}, k_{2} \sigma_{2}, S \rightarrow k_{3} \sigma_{3}, k_{4} \sigma_{4}, S^{\prime}\right)= \\
& \left\langle k_{3} \sigma_{3}, k_{4} \sigma_{4}, S^{\prime}\left|\hat{V} \frac{1}{\xi_{k_{1}}+\xi_{k_{2}}-\hat{H}_{0}} \hat{V}\right| k_{1} \sigma_{1}, k_{2} \sigma_{2}, S\right\rangle .
\end{aligned}
$$

The denominator in Eq. (3) is the energy of the intermediate virtual state, which equals $\pm\left(\xi_{k_{1}}-\xi_{k_{3}}\right)$ for two of the four possible pairings of the electron creationannihilation operators in Eqs. (2) and (3) (one of these pairings is shown on Fig. 1), or $\pm\left(\xi_{k_{1}}-\xi_{k_{4}}\right)$ for the other two pairings. The spin structure of the scattering amplitude can easily be found from Eq. (3). In a scattering event, spins of one or both participating electrons must flip, with the corresponding change of the impurity spin. In this paper we are interested only in the relaxation of the electron energy distribution, and assume that the system does not have any spin polarization. Therefore we need to calculate only the total cross-section of scattering into all possible spin states, averaged over the initial spin states of the impurity and two electrons. After this averaging, the terms proportional to $\left[\left(\xi_{k_{1}}-\xi_{k_{3}}\right)\left(\xi_{k_{1}}-\xi_{k_{4}}\right)\right]^{-1}$ drop out. Finally, we get the rate of scattering of two electrons with energies $\varepsilon_{1}$ and $\varepsilon_{2}$ into states with energies $\varepsilon_{3}$ and $\varepsilon_{4}$ :

$$
\Gamma\left(\varepsilon_{1}, \varepsilon_{2} ; \varepsilon_{3}, \varepsilon_{4}\right)=\frac{\pi}{4} \frac{n}{\nu} S(S+1)(J \nu)^{4}
$$

$$
\times\left[\frac{1}{\left(\varepsilon_{1}-\varepsilon_{3}\right)^{2}}+\frac{1}{\left(\varepsilon_{1}-\varepsilon_{4}\right)^{2}}\right] \delta\left(\varepsilon_{1}+\varepsilon_{2}-\varepsilon_{3}-\varepsilon_{4}\right) .
$$

Substituting this expression into the collision integral [9] and taking into account the symmetry $\varepsilon_{1} \leftrightarrow \varepsilon_{2}, \varepsilon_{3} \leftrightarrow \varepsilon_{4}$ of the right-hand side of Eq. (何), we obtain the collision integral in the form:

$$
\begin{aligned}
I(\varepsilon,\{f\}) & =\iint d E d \varepsilon^{\prime} K(E) \\
& \times\left\{f(\varepsilon) f\left(\varepsilon^{\prime}\right)[1-f(\varepsilon-E)]\left[1-f\left(\varepsilon^{\prime}+E\right)\right]\right. \\
& \left.-f(\varepsilon-E) f\left(\varepsilon^{\prime}+E\right)[1-f(\varepsilon)]\left[1-f\left(\varepsilon^{\prime}\right)\right]\right\}
\end{aligned}
$$

where the kernel

$$
K(E)=\frac{\pi}{2} \frac{n}{\nu} S(S+1)(J \nu)^{4} \frac{1}{E^{2}}
$$

depends only on the energy $E$ transferred in the collision, and $f(\varepsilon)$ is the electron energy distribution function.

The above derivation of the inelastic amplitude [Eq. (3)] was performed in the lowest non-vanishing order of the perturbation theory. It is known from the theory of the Kondo effect, that for the elastic scattering amplitude, the calculation in the lowest order may be insufficient. The higher in $J \nu$ orders yield contributions to the elastic scattering which logarithmically diverge at low energies 3.,8]. For elastic scattering, the leading terms in all orders can be summed up with the help of the renormalization-group technique [10]. In this technique, the bare exchange constant is replaced by the renormalized one,

$$
J(\varepsilon)=\left[\nu \ln \left(|\varepsilon| / T_{K}\right)\right]^{-1},
$$

and the scattering amplitude at energy $\varepsilon$ is to be calculated within the Born approximation in $J(\varepsilon)$. The Kondo temperature $T_{K}$ is related to the parameters of the Hamiltonian (1) by

$$
T_{K}=\mu \sqrt{J \nu} D \exp (-1 / J \nu)
$$

Here $D$ is the high-energy cut-off, and $\mu \sim 1$ (for detailed discussion of these parameters, see Ref. [11]).

Similar to the theory of elastic scattering, the lowestorder result (6) is valid only while $\ln \left(\left|\varepsilon_{i}\right| / T_{K}\right) \gg 1$. At smaller energies, the two vertices of Fig. 1 acquire corrections of the form $J \nu\left[J \nu \ln \left(D /\left|\varepsilon_{i}\right|\right)\right]^{m}$ in the $(m+1)$-st order of the perturbation theory. The two vertices are renormalized independently from each other [12], and the corresponding diagrams have the same structure as in 
Ref. 87. This approximation is justified as long as the energies $\varepsilon_{i}$ of all incoming and outgoing electrons satisfy the condition

$$
\ln \left(\left|\varepsilon_{i}\right| / T_{K}\right) \gtrsim 1
$$

The resulting non-perturbative expression for the kernel in the collision integral reads

$$
\begin{aligned}
K\left(E ; \varepsilon, \varepsilon^{\prime}\right) & =8 \pi \frac{n}{\nu} S(S+1)\left[\ln \frac{|\varepsilon|}{T_{K}}+\ln \frac{|\varepsilon-E|}{T_{K}}\right]^{-2} \\
& \times\left[\ln \frac{\left|\varepsilon^{\prime}\right|}{T_{K}}+\ln \frac{|\varepsilon+E|}{T_{K}}\right]^{-2} \frac{1}{E^{2}}
\end{aligned}
$$

We would like to emphasize that the dependence of the kernel $K$ on the energies of participating electrons remains weak (logarithmic), as opposed to the strong $1 / E^{2}$ dependence on the transferred energy $E$. The logarithmic dependence in Eq. (10) is meaningful as long as the electron energies $\varepsilon_{i}$ exceed the smearing of the Fermi level caused by temperature or a non-equilibrium electron distribution. In the opposite case, the arguments of all the logarithms in Eq. (10) must be replaced by $\varepsilon^{*} / T_{K}$, where energy $\varepsilon^{*}$ characterizes the smearing. It is important to note that $\varepsilon^{*}$ does not cut off the singularity in the transferred energy $E$.

The low-energy divergence of the scattering rate (4) stems from the degeneracy of spin states of the impurity. Due to this degeneracy, the energy of the intermediate virtual state [the denominator in Eq. (3)] approaches zero at $E \rightarrow 0$. An additional condition for the divergency is the time independence of the average $\left\langle S^{\prime}\left|\hat{S}^{j}(t) \hat{S}^{k}\left(t^{\prime}\right)\right| S\right\rangle$ in the approximation of Eq. (2) (here $j, k=x, y, z)$. In fact, exchange interaction between the itinerant electrons and impurity may flip its spin. The resulting impurity spin correlation function decays, restricting the lifetime of the intermediate virtual state. The corresponding decay rate cuts off the $E=0$ singularity of the kernel 10 . The manner of decay depends on the electron energy distribution $f(\varepsilon)$. We will discuss first the cut-off in the case of weak deviations of $f(\varepsilon)$ from the thermal equilibrium.

Let us first consider the low-energy cut-off for $K(E)$ at high temperatures, $T \gg T_{K}$. Scattering of electrons off the spin results in exponential decay of the correlation function, $\left\langle S^{\prime}\left|\hat{S}^{j}(t) \hat{S}^{k}\left(t^{\prime}\right)\right| S\right\rangle \propto \exp \left(-\left|t-t^{\prime}\right| / \tau_{T}\right)$. The impurity spin correlation time $\tau_{T}$ can be evaluated with the help of the Fermi golden rule. Since the deviation from the thermal equilibrium is weak, we can replace $f(\varepsilon)$ with the Fermi distribution function $n_{F}(\varepsilon)$,

$$
\begin{aligned}
\frac{\hbar}{\tau_{T}} & =\frac{2 \pi}{3} S(S+1)(J \nu)^{2} \int d \varepsilon n_{F}(\varepsilon)\left[1-n_{F}(\varepsilon)\right] \\
& =\frac{2 \pi}{3} S(S+1)(J \nu)^{2} T
\end{aligned}
$$

When $T$ is lowered towards $T_{K}$, the exchange constant in Eq. (11) is renormalized according to Eq. (7) with $\varepsilon=T$. The resulting expression for the spin-flip rate reads:

$$
\frac{\hbar}{\tau_{T}}=\frac{2 \pi}{3} S(S+1)\left[\ln \left(T / T_{K}\right)\right]^{-2} T .
$$

The energy scale $\hbar / \tau_{T}$ sets the limit of applicability of Eq. (3) and cuts off the singularity in the kernel (10) at $E \sim \hbar / \tau_{T}$. Note that within the limits of applicability of Eq. (12), the spin-flip rate satisfies the condition $T>\hbar / \tau_{T}>T_{K}$.

At $T<T_{K}$, we can neglect the effect of a finite temperature on the scattering of electrons with $\left|\varepsilon_{i}\right| \gg T_{K}$. The behavior of $K(E)$ can be related to the time dependence of the zero-temperature self-correlation function of the impurity spin. At time difference $\left|t-t^{\prime}\right| \gg \hbar / T_{K}$ this correlation function decays as $\left\langle G S\left|\hat{S}^{j}(t) \hat{S}^{k}\left(t^{\prime}\right)\right| G S\right\rangle \sim$ $\delta_{j k} /\left[T_{K}\left|t-t^{\prime}\right|\right]^{2}$, and therefore the singularity of the kernel $K(E)$ is cut off at $E \sim T_{K}$ (here $|G S\rangle$ is the ground state of the Kondo problem).

At this point, we should mention that at energy transfers $E \sim T_{K}$, the processes with participation of three or more electrons must also be taken into account along with the two-particle scattering. The consideration of these multi-particle processes is an arduous task lying beyond the scope of this paper. Here we address mostly the electron energy relaxation on large $\left(\gtrsim T_{K}\right)$ energy scales. It allows us to limit our consideration to the twoparticle processes accounted for by Eq. (5), and dispense with the scattering events involving more particles.

At very small energies $\left(\left|\varepsilon_{i}\right|, T \ll T_{K}\right)$, however, the Fermi-liquid description of electrons is again a valid tool. The behavior of the system is described in this case by the quadratic fixed-point Hamiltonian, in which the fourfermion interaction is a least-irrelevant term [13,14]. The calculation of the inelastic scattering rate is then straightforward, the resulting collision-integral kernel is given by

$$
K(E)=\frac{1}{T_{K}^{2}} \frac{n}{\nu}
$$

When $T=0$, the corresponding rate of inelastic electron scattering is $\hbar / \tau_{\text {in }}=\int_{0}^{\varepsilon} d E K(E) E \propto\left(\varepsilon / T_{K}\right)^{2}$. At $\varepsilon \rightarrow 0$, it decreases faster than $\varepsilon$, as it is supposed to be in the Fermi-liquid picture.

Relaxation of the electron energy distribution was investigated experimentally in metallic wires of $\mathrm{Cu}$ and $\mathrm{Au}$ in Refs. [4.5]. In these experiments, a finite bias $V=50-500 \mu \mathrm{eV}$ was applied to the ends of a wire. It was found that starting from fairly small wire lengths, the electron distribution is smeared over the range of energies $\mathrm{eV}$, instead of having two distinct steps created by the bias applied to the wire ends. The observed electron energy relaxation was attributed [4.50 to two-electron collisions. The collision-integral kernel for $E<e V$ extracted from the experiments has the form $K(E)=\hbar /\left(\tau_{0} E^{2}\right)$, with a cut-off at some low energy, which scales linearly 
with $\mathrm{eV}$ 15. The value of the parameter $\tau_{0}$ was $0.5-1.0$ $\mathrm{ns}$ for $\mathrm{Cu}$ wires, and $0.1 \mathrm{~ns}$ for $\mathrm{Au}$ wires 16 .

Now we discuss the possibility of such relaxation due to the electron scattering on magnetic impurities in wires. In the experimental setup, the electron distribution is smeared, and the width of smearing $\mathrm{eV}$ exceeds both $T$ and the energies $\left|\varepsilon_{i}\right|$ of the colliding electrons. Assuming also that $e V \gg T_{K}$, we can simplify the kernel (10):

$$
K(E)=\frac{\pi}{2} \frac{n}{\nu} S(S+1)\left[\ln \left(e V / T_{K}\right)\right]^{-4} \frac{1}{E^{2}} .
$$

The $1 / E^{2}$ dependence in Eq. (14) persists down to the cut-off, which is determined by the spin-flip rate $1 / \tau_{e V}$. The derivation of the spin-flip rate in these nonequilibrium conditions follows the same path which led to Eq. (12), and results in:

$$
\frac{\hbar}{\tau_{e V}}=\gamma S(S+1)\left[\ln \left(e V / T_{K}\right)\right]^{-2} e V .
$$

Here the numerical constant $\gamma \sim 1$ depends on the detailed shape of the non-equilibrium electron distribution.

Now we compare theoretical results (14) and (15) with the experimental observations for $\mathrm{Au}$ wires [5]. Properties of these samples are compatible with the presence of iron impurities with a concentration up to few tens of ppm 15. We take the density of states in Au at the Fermi level $\nu \approx 0.25(\mathrm{eV} \text { site })^{-1}$ [17], and $T_{K} \approx 0.3 \mathrm{~K}$ for Fe impurities in $\mathrm{Au}$ [2]. The typical value of voltage in the experiments [5] was $V \approx 0.3 \mathrm{meV}$ [15]. Substituting these parameters into Eq. (14), we see that a relatively small concentration $n \sim 10 \mathrm{ppm}$ is sufficient to reproduce the experimentally measured value $\tau_{0} \approx 0.1 \mathrm{~ns}[18$. The spin-flip rate (15) is the low-energy cut-off for the $1 / E^{2}$ dependence of the kernel. This cut-off is roughly proportional to the applied voltage, in agreement with experimental observations [15. We must note, however, that the lower voltages used in experiment [5] are close to the Kondo temperature, so the leading-logarithmic approximation [8,10], used in derivation of Eqs. (14)-(15), may be insufficient.

The inelastic electron scattering off magnetic impurities must be sensitive to an external magnetic field polarizing the spins in the system. The Zeeman splitting $g \mu H$ of the spin states prevents the impurity spins from changing, thus suppressing the inelastic scattering processes with energy transfers $E<g \mu H$. Measurements in a sufficiently strong magnetic field may elucidate the role of magnetic impurities in the electron energy relaxation.

An important feature of the electron-electron interaction mediated by magnetic impurities is that it is not translationally invariant. This is why the introduction of non-magnetic impurities, which affects drastically Coulomb interaction of electrons [6], produces only small corrections to the interaction induced by magnetic impurities.
The above consideration was performed for the magnetic impurities described by the one-channel Kondo model. However, the proportionally of the scattering integral kernel to $1 / E^{2}$ at $E \gg T_{K}$ holds for an arbitrary number $N$ of channels in the Kondo problem. If the exchange constants $J$ are the same for all channels, the kernel (6) acquires an additional factor $N^{2}$. For the specific case $N=2$, the qualitative $K(E) \propto 1 / E^{2}$ behavior was noticed in Ref. [19]. However in Ref. 19] this behavior was attributed solely to $N=2$; this, in our opinion, is inaccurate. Our consideration also indicates that at any $N$, the $1 / E^{2}$ divergence of the kernel $K(E)$ is cutoff at small $E$, see Eq. (15). This is also in an apparent disagreement with Ref. [19], which states that for $N=2$ the divergence persists down to the lowest energies.

In conclusion, we have shown that the exchange interaction of itinerant electrons with magnetic impurities can facilitate inelastic electron-electron scattering. We derived the kernel of the corresponding collision integral, and found its explicit dependence on the parameters of the system for a wide range of the energies of colliding electrons. This allowed us to perform a quantitative analysis of the experimental results of Refs. [4.5]. We find that a very small density of magnetic impurities could lead to the anomalies in the electron energy relaxation observed there.

This work was supported by NSF Grants No. DMR 97-31756 and DMR 98-12340. A. K. is a recipient of University of Minnesota's Doctoral Dissertation Fellowship. The authors are grateful to I. Aleiner, N. Birge, D. Esteve, A. Ludwig and H. Pothier for valuable discussions.

[1] B.L. Altshuler et al., Sov. Phys. JETP 54, 411 (1980).

[2] A.C. Hewson, The Kondo problem to heavy fermions (Cambridge University Press, 1993).

[3] J. Kondo, Prog. Theor. Phys. 32.37 (1964).

[4] H. Pothier et al., Phys. Rev. Lett. 79, 3490 (1997).

[5] F. Pierre et al., cond-mat/0012038 and in: Proceedings of the NATO Advanced Research Workshop on sizedependent magnetic scattering, Pecs, Hungary, 2000, in press.

[6] B.L. Altshuler, A.G. Aronov, in Electron-electron interactions in disordered systems, ed. by A.L. Efros and M. Pollak (North-Holland, Amsterdam, 1985).

[7] J. Sólyom, A. Zawadowski, Z. Physik 226, 116 (1969).

[8] A.A. Abrikosov, Physics 2, 21 (1965).

[9] A.A. Abrikosov, Fundamentals of the theory of metals (New York, Elsevier, 1988), p. 57.

[10] P.W. Anderson, J. Phys. C 3, 2436 (1970).

[11] F.D.M. Haldane, J. Phys. C 11, 5015 (1978).

[12] Diagrams with more than one spin line connecting the two electron lines of Fig. 1 either yield zero after summa- 
tion over the spin indices of intermediate states, or are proportional to a lower power of the logarithmic factor.

[13] P. Nozières, J. Low Temp. Phys 17, 31 (1974).

[14] I. Affleck and A.W.W. Ludwig, Phys. Rev. B 48, 7297 (1993).

[15] H. Pothier, private communication.

[16] A.B. Gougam et al., J. Low Temp. Phys. 118, 447 (2000).
[17] C. Kittel, Introduction to solid state physics (John Wiley \& sons, New York, 1996).

[18] In the framework of our model, the electron dephasing time is given by $\tau_{\phi} \sim \tau_{0} / \ln ^{2}\left(e V / T_{K}\right)$. The value $n \sim 10$ ppm yields the estimate $\tau_{\phi} \sim 10 \mathrm{ps}$, in agreement with the experiments [5].

[19] J. Kroha, Adv. Solid State Phys. 40, 267 (2000). 